\section{Manufacturing and Processing Techniques Affecting Morphology of Pyrotechnic Oxidizer Particles}

Richard C. Dujay, Mesa State College EM Microscopy Facility Ken Kosanke, PyroLabs, Inc.

In a pyrotechnic composition, the morphology (size, shape and surface features) of individual particles in the mixture influences both burn rate and the ease of ignition of the composition. Collectively, the larger, more rounded and smoother the particles, the lower the burn rate and the more difficult it is to ignite the composition. However, the purpose of the current investigation was only to catalogue some particle morphologies that may be useful in forensic identification of unconsumed or residual pyrotechnic evidence. In this study, potassium nitrate was chosen because of its common use in pyrotechnics and because of its similarity with other frequently used pyrotechnic oxidizers.

All samples were prepared for scanning electron microscopy using a double-sided adhesive carbon disk applied to an aluminum post. Particles were mounted to the carbon disk and sputter coated with gold. Examination of the particles was performed at Mesa State College using a newly remanufactured
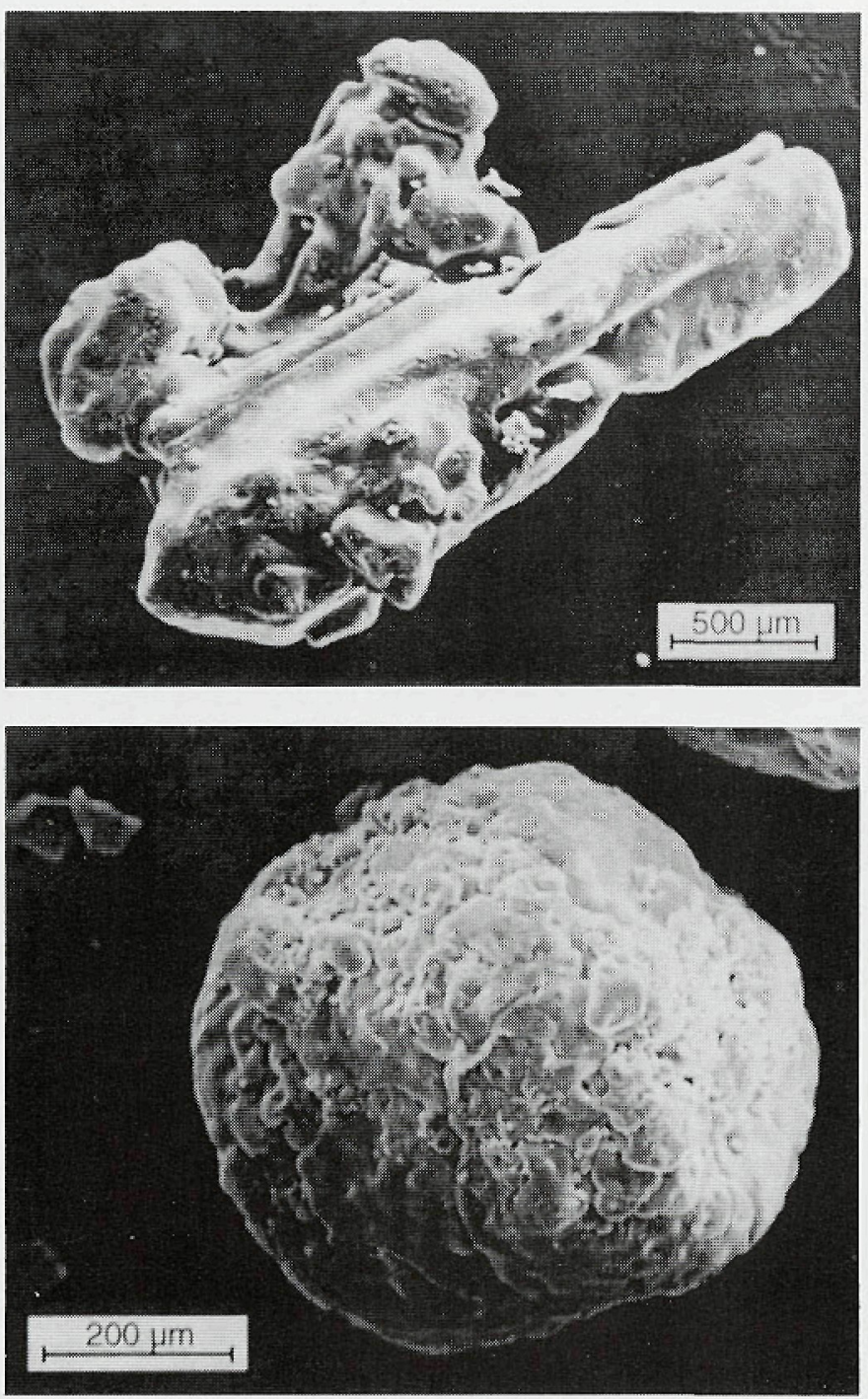

Figure 1. Somewhat typical examples of the AR (upper) and AgP (lower) grade potassium nitrate particles. Upper, $35 \times$ magnification, lower, 100x magnification.

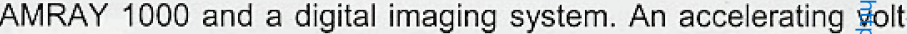
age of $20 \mathrm{kV}$ was used with a final aperture diameter of $200 . \mathrm{jm}$ The working distance was approximately $12 \mathrm{~mm}$.

The primary source of potassium nitrate investigated was analytical reagent (AR) grade with an initial particle size of $2 \overrightarrow{50}$ 3400 microns. Another source was an agricultural prill (AgP) with an initial particle size of 100-1000 microns. The AR grade particles initially presented numerous linear structures, whereas the AgP presented mainly spherical structures, see Figure 1.

Material of this size is much too coarse to be useful in porrotechnics and must be reduced in size. One common methoof o particle size reduction is by crushing or grinding. Often this istac complished using a rotating disk mill similar to a grain mill, utilizing either metal or stone disks. The particles shown in Figure 2 . the result of such milling. While the goal was to reduce the parti cles to a size of less than approximately 300 microns, nume particles smaller than 20 microns are also produced-seen laere adhering to the surface of the larger particle. (Quite similar pearing particles are also produced by crushing, using a siro:ple mortar and pestle.)

Another common method of particle size reduction is to
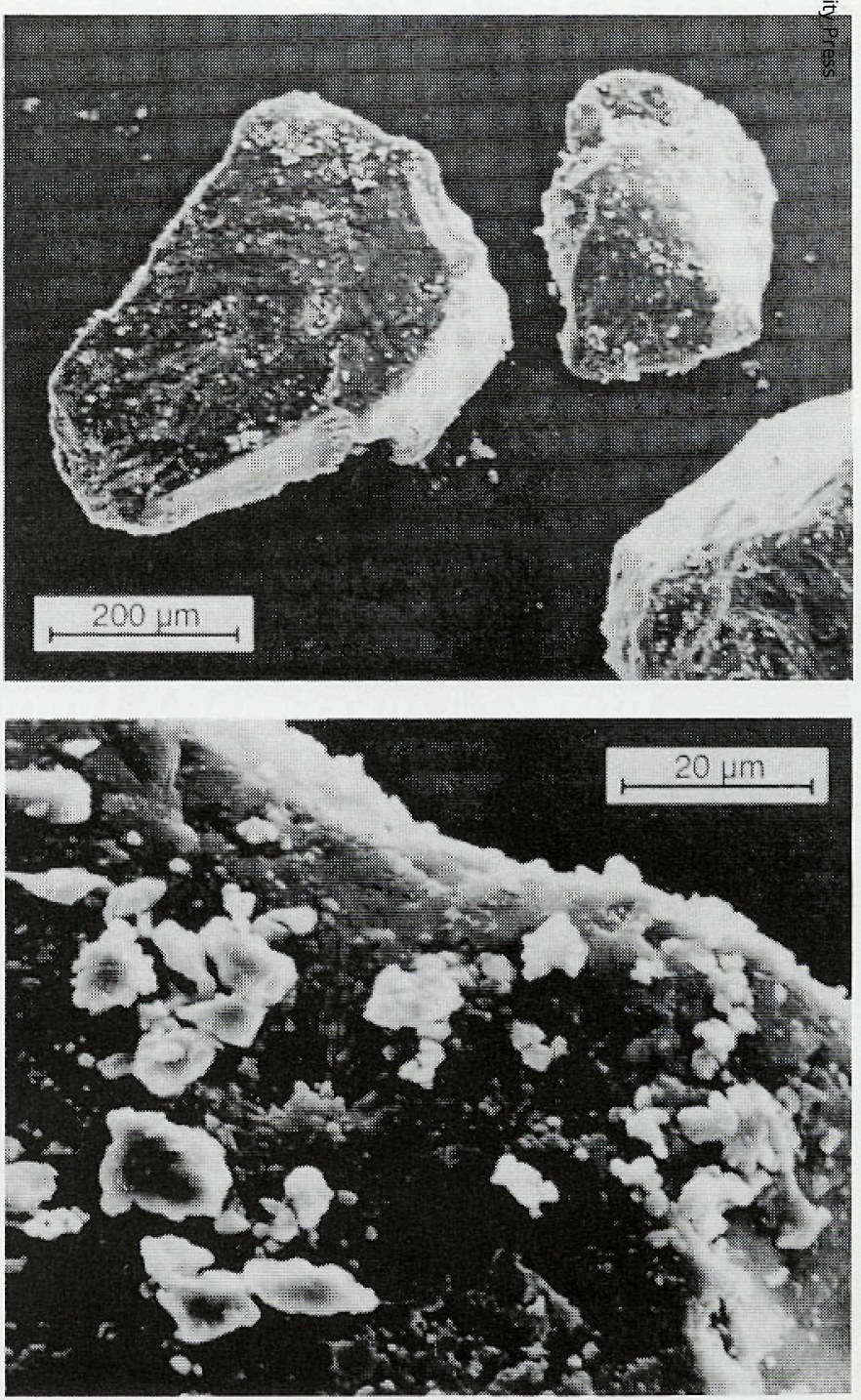

Figure 2. Two images of AR grade potassium nitrate, 60 to 100 mesh particles produced using a rotating disk mill, documenting the presence of many tiny particles adhering to the surfaces of larger particles. Upper, $100 \times$ magnification, lower $1000 \times$ magnification.

Continued on page 10 


\section{BEFORE}

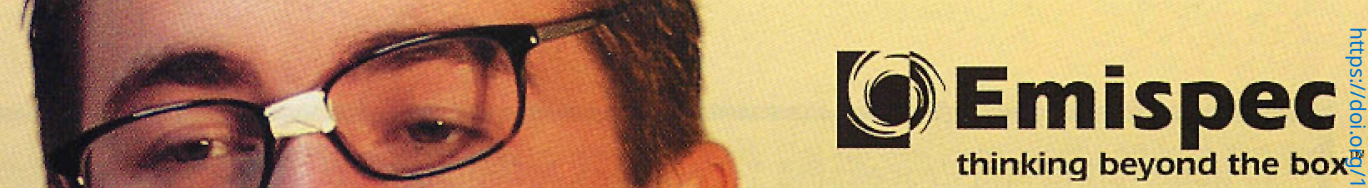

(8)

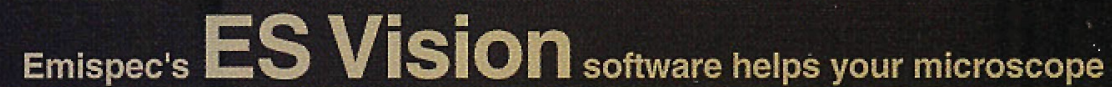

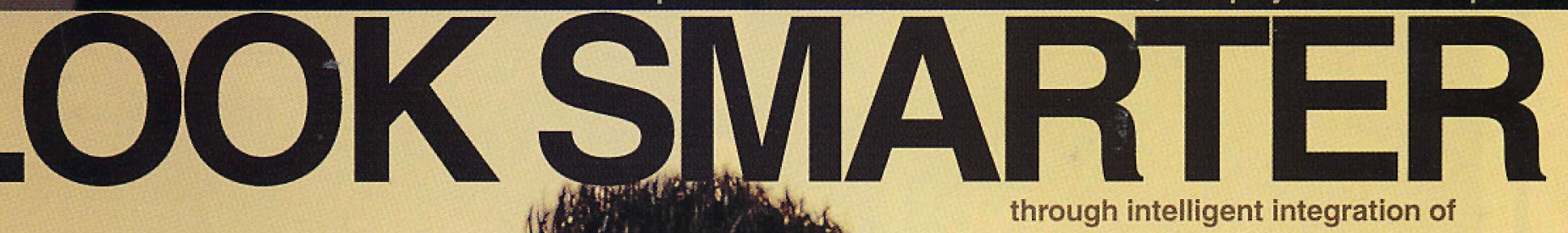

through intelligent integration of detectors. Your IQ won't get any higher, but the speed and accuracy of your results will convince your colleagues that it has.

To learn more, visit www.emispec.com/looksmarter

AFTER 
Manufacturing and Processing Techniques Affecting Morphology of Pyrotechnic Oxidizer Particles Continued from page 8

ploy the use of a ball mill. In this case, the ball mill consisted of a rotating drum containing ceramic cylinders $(12 \mathrm{~mm}$ in diameter by $12 \mathrm{~mm}$ in length). The result of milling the potassium nitrate for 12 hours is presented in Figure 3 . Although most of the individual particles are significantly smaller than $40 \mathrm{mi}-$ crons, there was a tendency for them to clump or fuse together creating much larger particles. This was due to slight residual moisture in the AR grade potassium nitrate; thorough drying before processing greatly reduces this tendency to agglomerate.

The shape of the particles after processing tended to fall into two distinct categories, those with sharp angular features (Figure 2) and those with rounded margins (Figure 3 ). These categories (sharp and rounded) can be helpful in identifying processing techniques of the materials used in manufacturing pyrotechnic explosive devices. However, perhaps more importantly, such characterization can be useful in establishing a forensic match between known and evidentiary materials.

A third method of particle size reduction-coacervation-
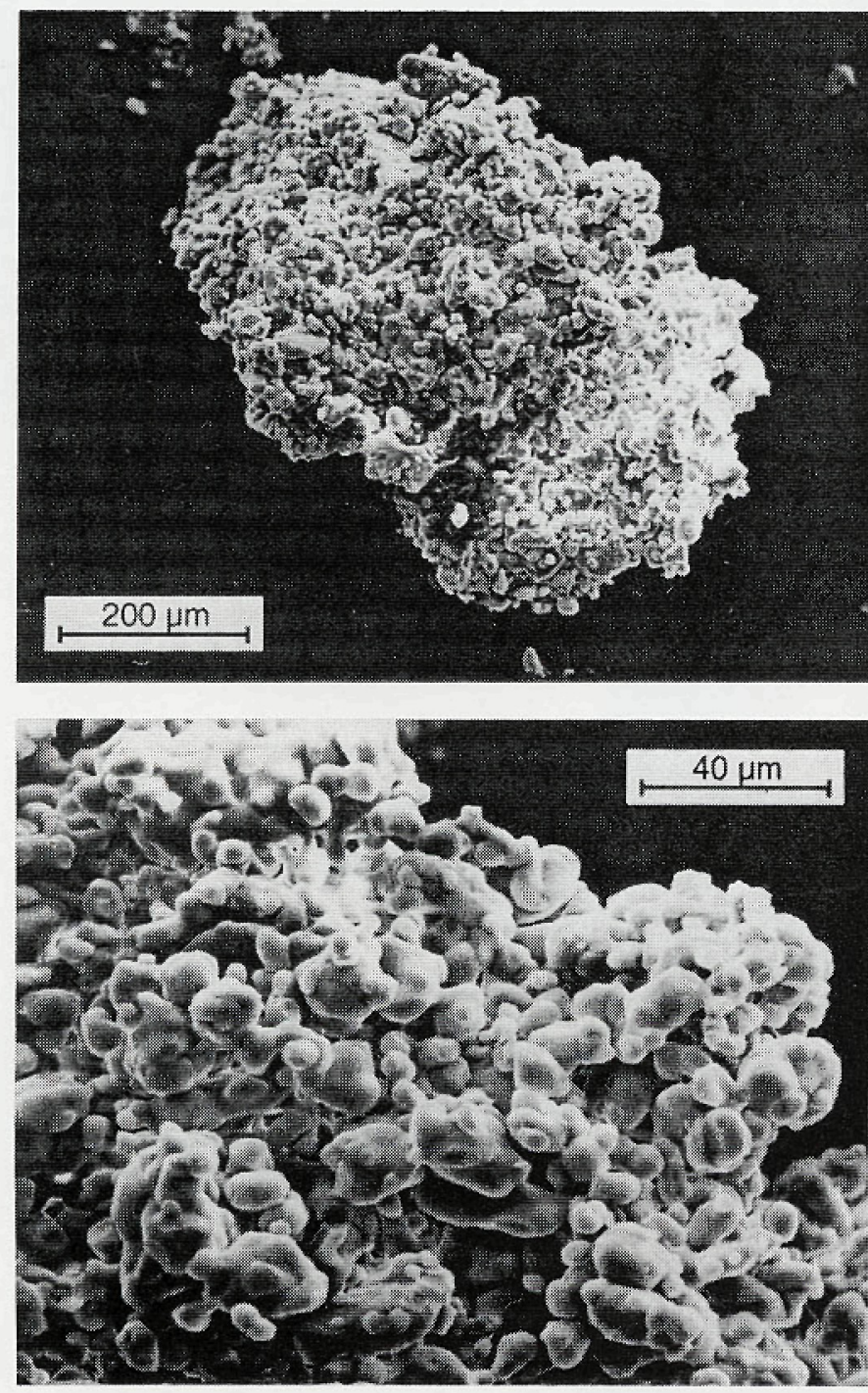

Figure 3. Two mesh fractions of undried AR grade potassium nitrate produced by ball milling for 12 hours. Upper, a particle in the range of 60 to 100 mesh at $100 \mathrm{x}$ magnification, lower, the same particle at $500 \mathrm{x}$ magnification. has gained some popularity among individuals because it doe: not require machinery. In this case, potassium nitrate is dissofve in water to create a nearly saturated solution; this is often formed while heating the solution to expedite the process and in crease the amount of material in solution. Once the material is i solution, it is removed from the heat source and alcohol is quïckl added while stirring rapidly. The potassium nitrate is forced oo to solution forming particles of a much smaller particle size thanth original material. Their appearance is quite distinct from the ofthe methods and is illustrated in Figure 4.

The last method of particle size reduction to be discuss hammer milling, where high-speed metal blades are used to re duce the size of the material. These blades shatter the potas giun nitrate crystals and produce much more noticeable fracture fopat terns than crushing or ball milling. Figure 5 illustrates the exen sive fracture patterns and whiskering (the more or less pagälle ridges on the fractured surface of the crystals).

There are other surface features that may aid in identifin the processing method or in establishing a forensic match. ฆै first are pits or divots on the surface of larger particles sucti a seen in Figure 6 . These particles were produced by ball milling
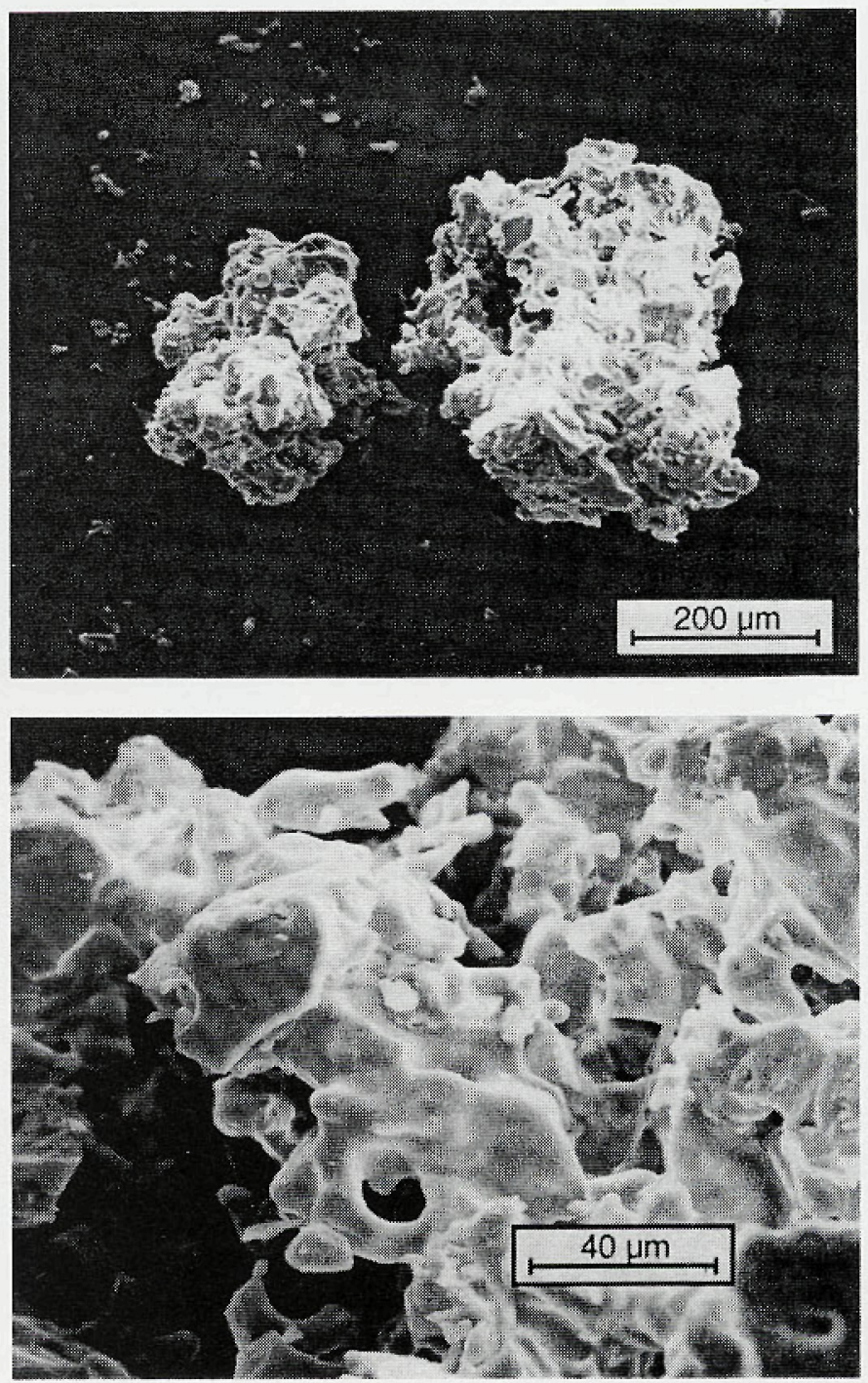

Figure 4. Particles from two mesh fractions of AR grade potassium nitrat produced by hot coacervation with rapid stirring. Upper, 60 to 100 mes particles at $400 \times$ magnification; lower, a portion of the particle on the righ at $500 \times$ magnification. 


\section{An Integrated System Designed for High Speed System Automation}

Universal Imaging is pleased to announce the release of our new hardware platform, the Genll Multi Dimensional Imager (MDl).

Originally developed for High Throughput Screening applications, the MDI is now available through our direct and dealer sales network.

The new Multi Dimensional Imager is the first fully automated system designed from the ground up to provide high performance for demanding imaging applications requiring complete system automation.

Uses MetaMorph ${ }^{8}$ software as the Engine

The MDI utilizes the industry-standard MetaMorph ${ }^{\circledR}$ engine and is designed for virtually any multi-dimensional application.

Some of the new features include:

- 10 position excitation filter wheel

- 10 position emission filter wheel

- 10 position neutral density filter wheel

- 5 position dichroic filter wheel

- $175 \mathrm{~W}$ Xenon light source

- High Speed focus device

- Automated 6-position objective turret

- Motorized precision stage

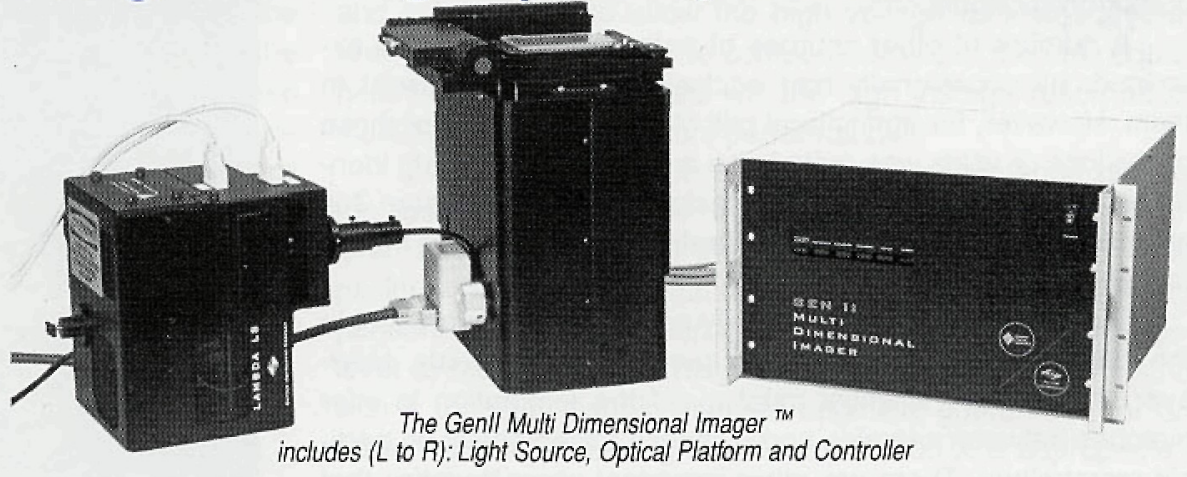

The heart of the system contains the highspeed dichroic wheel coupled with the flexibility of the 10 position excitation and emission filter wheels.

All filter wheels operate simultaneously, which permits rapid, easy changes for optimization of the system to the selected fluorophore. This provides the shortest possible exposure times with optimal light output. The high-speed focus motor operates in as little as $30 \mathrm{msec}$ and permits rapid Z-series data collection while maintaining the proper focus for

\section{all selected objectives.}

The user may choose any camera currently supported by the MetaMorph Imaging System and add filter sets appropriate to their specific application.

Acquisition, Analysis Modules for Automation

We've created new unique software acquisition and analysis modules which fully automate the hardware configurations, thereby streamlining and simplifying these processes for the end user.

\section{New MetaMorph ${ }^{\circledR}$ Imaging System Includes Features for Entry Level and Advanced Users}

Universal Imaging Corporation has released a full upgrade of its market leading MetaMorph $^{(8)}$ Imaging System.

The newly redesigned Version 4.6 includes a streamlined interface for greater ease of use, an interactive training $C D$ and powerful new functions for advanced image acquisition, processing and analysis.

To make live cell imaging more accessible to new users, the MetaMorph ${ }^{\circledR}$ system now features application-specific toolbars such as the Fluorescence Tools for acquiring multiple wavelengths from digital cameras, measuring colocalization between probes and overlaying images.

The simplified interface of

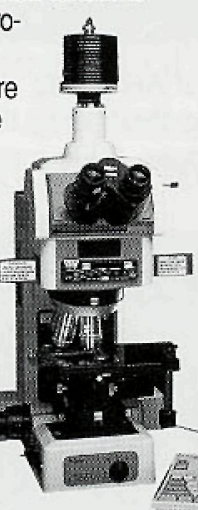
MetaMorph ${ }^{\ominus}$ Imaging System

can label regions on the image with numbers, data or other text they define.

The advanced features added to commands for removing haze from image planes and new functions for High Content Screening. The new HCS Acquire function coordinates control of the stage, the illumination devices and camera hardware. After acquisition, the HCS Viewer
organizes large quantities of image data and measurements into an informative display for easy analysis.

The training $\mathrm{CD}$ gives users an animated tour of the software and describes the most common MetaMorph ${ }^{(B)} 4.6$ makes image acquisition much easier. The Acquire dialog box includes commands for setting the camera binning, gain and auto-exposure. It also enables users to control camera shutters during acquisition, set exposure times and acquisition regions, and more. Users can expand the interface to access advanced settings such as background subtraction and shading correction. is a snap with the new Region Measurements function, which combines intensity, area, and other parameters in one easy-to-use interface. Users

Windows ${ }^{\circledR} 95 / 98 / 2000$ and NT, with support for a variety of black \& white and color cooled CCD cameras from major manufacturers. Available software drivers support automated microscopes, filter wheels and shutters, monochromators, focus motors and Piezo electric focus devices, motorized stages, robotic peripherals and any digital or serial input/output device. MetaMorph ${ }^{\circledR} 4.6$ include two deconvolution

Quantifying gray values in a biological sample

\section{The Biolmaging Leader}

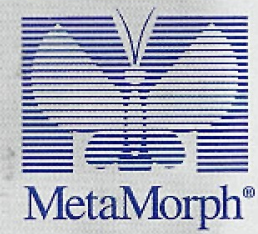

Software and Systems for:

6-D Imaging

- Brightness Measurements

- Colocalization

- Fluorescence Recovery After Photobleaching (FRAP)

- Fluorescence Resonance Energy Transfer (FRET)

- Gene Chips \& Microarrays

- Green Fluorescent Protein

- Intracellular Calcium, pH \& Magnesium

- Immunofluorescence

- Live/Dead Assays

- Motion Analysis

Morphometry

- Ratio Imaging

- Time Lapse

And Many More Applications...

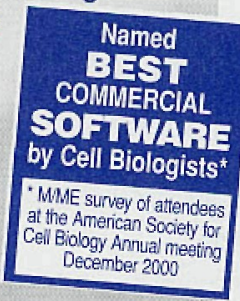

Working to Improve Your Image

UNIVERSAL IMAGING CORPORATION Downinglown, PA 19335 sales@universal-imaging.com 610-873-5610

Contact us for a new free brochure

www.universal-imaging.com 


\section{Manufacturing and Processing Techniques Affecting Morphology of Pyrotechnic Oxidizer Particles Continued from page 10}

but for only 2 hours.

A number of other sources of potassium nitrate were examined; all occasionally had occlusions or voids present in them. However, for agricultural prill (AgP), the number of these occlusions or voids was quite large and can serve to help identify the source or be used to help establish a match. Figure 7 is an example of $\mathrm{AgP}$ potassium nitrate crushed using a mortar and pestle.

The use of particle morphology can be an important tool in the identification and matching of pyrotechnic materials. However, the forensic scientist must avoid the temptation to infer matches between samples and evidence based solely on particle morphology. There are other important characteristics that should be considered, such as trace chemical composition. There are also differences in crystalline structure that can produce different morphologies for the same chemical processed using the same method.
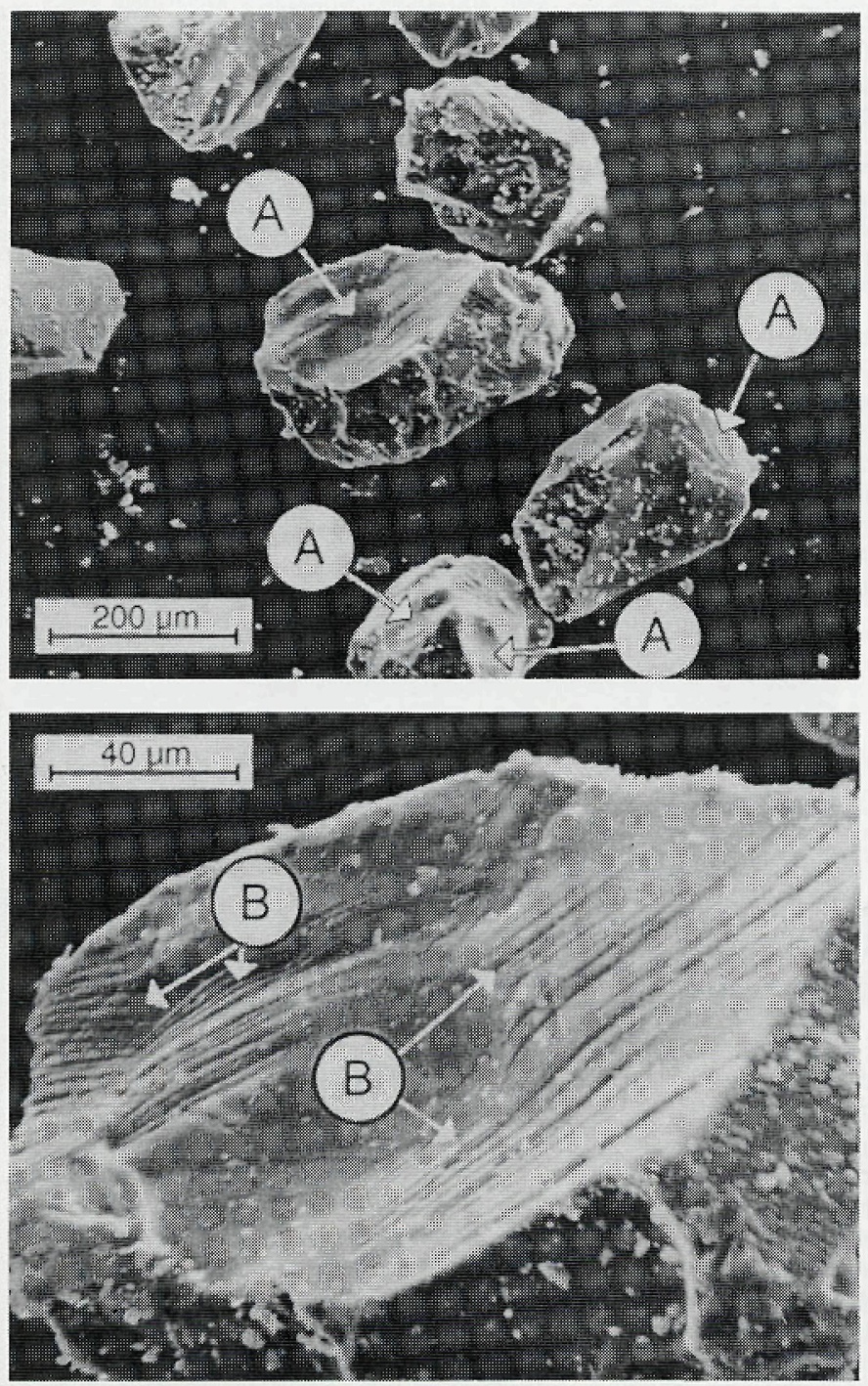

Figure 5. Particles of AR grade potassium nitrate produced by hammer milling, demonstrating fracture parerns $(A)$ and a rather extreme number of "whiskers" (B). Upper, 100x magnification, lower, 500x magnification.
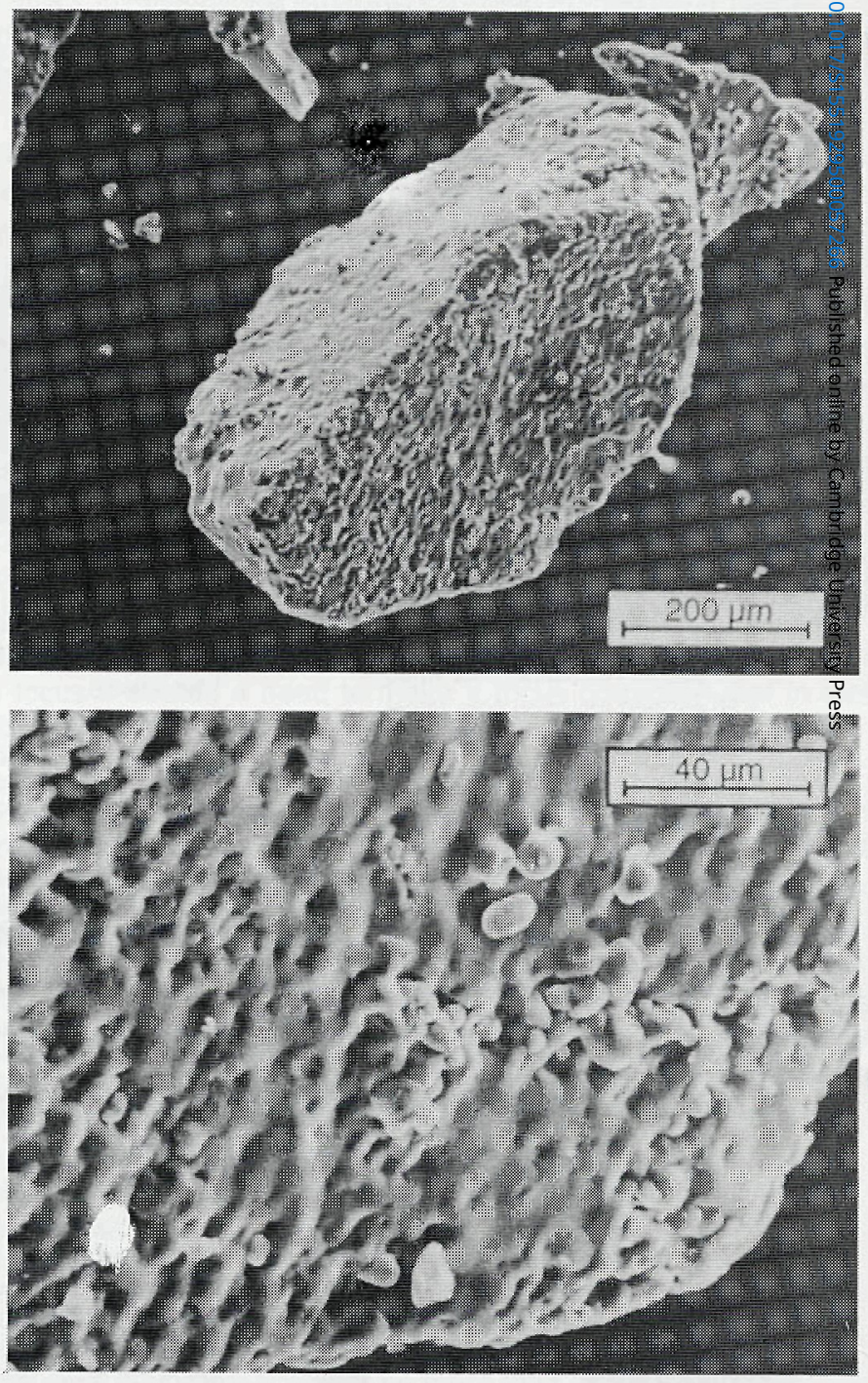

Figure 6. Particle of AR grade potassium nitrate produced by bal milling for 2 hours, illustrating the pitted surface of the 60 to 100 mesh particles, Upper, 100x magnification, lower, 500x magnifica. tion.

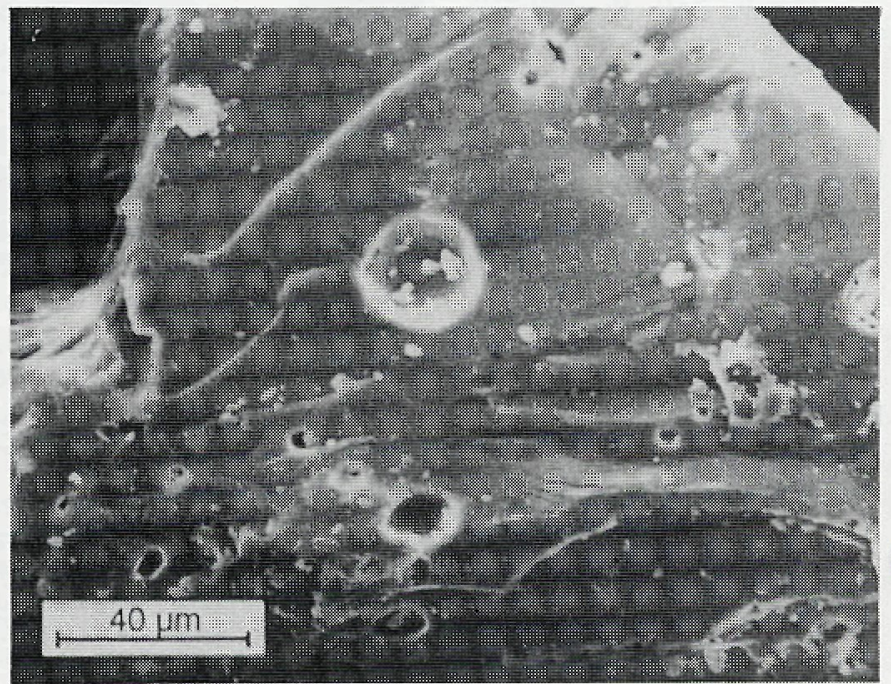

Figure 7. Particle of AgP grade potassium nitrate produced using a mortar and pestle, illustrating the presence of numerous occlusions, 500x magnification. 
Internet focussed solutions, streaming video, high resolution digital images. It's all here.......

\section{DUALVISION 600}
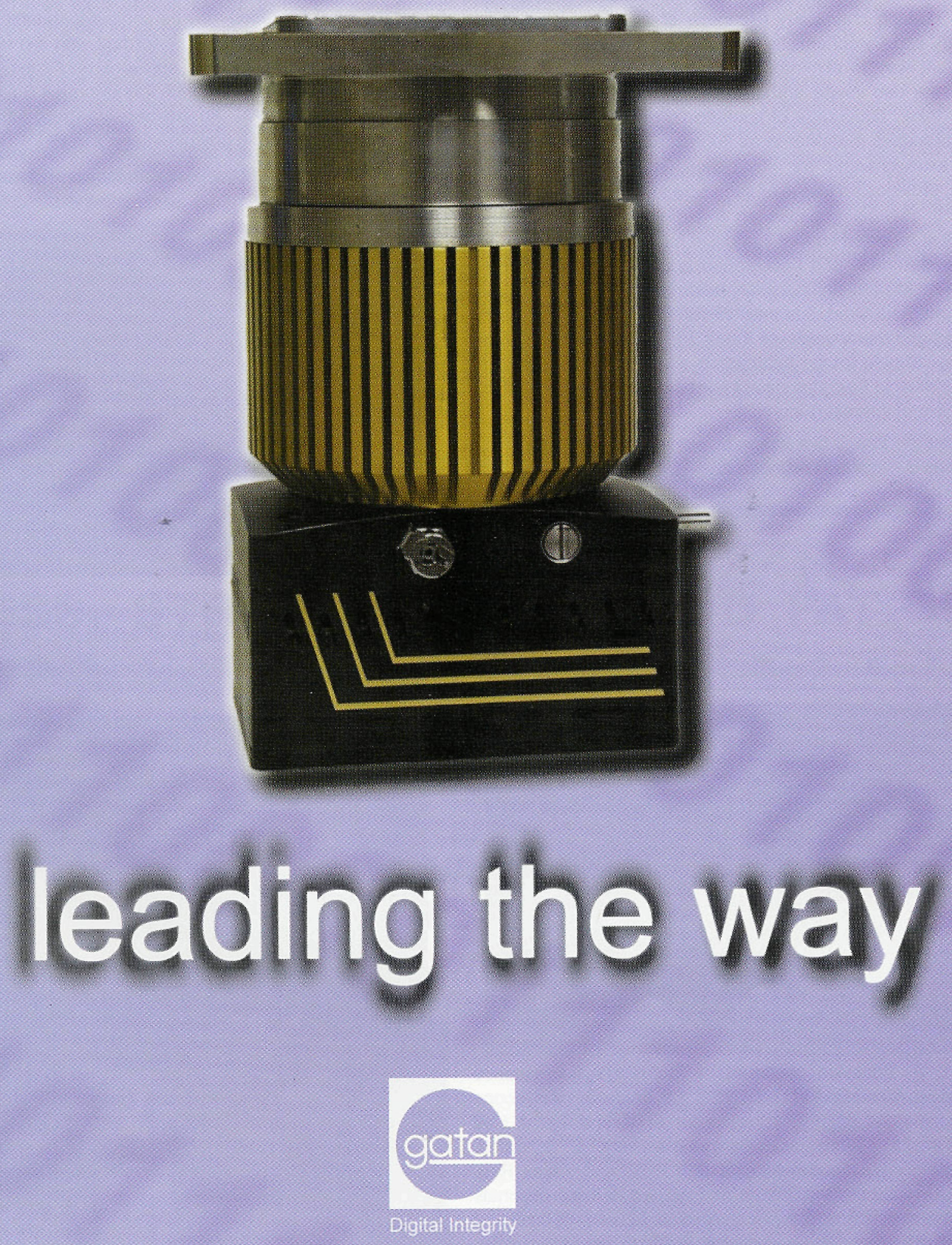